\title{
Fluoride complexation of rutherfordium (Rf, element 104)
}

\author{
By E. Strub ${ }^{1}$, J. V. Kratz ${ }^{*, 1}$, A. Kronenberg ${ }^{1}$, A. Nähler ${ }^{1}$, P. Thörle ${ }^{1}$, S. Zauner ${ }^{1}$, W. Brüchle ${ }^{2}$, E. Jäger ${ }^{2}$, M. Schädel $^{2}$, \\ B. Schausten ${ }^{2}$, E. Schimpf ${ }^{2}$, Li Zongwei ${ }^{2}$, U. Kirbach ${ }^{3}$, D. Schumann ${ }^{3}$, D. Jost ${ }^{4}$, A. Türler ${ }^{4}$, M. Asai ${ }^{5}$, Y. Nagame ${ }^{5}$, \\ M. Sakama ${ }^{5}$, K. Tsukada ${ }^{5}$, H. W. Gäggeler ${ }^{6}$ and J. P. Glatz ${ }^{7}$ \\ ${ }^{1}$ Institut für Kernchemie, Universität Mainz, D-55099 Mainz, Germany \\ 2 Gesellschaft für Schwerionenforschung, D-64291 Darmstadt, Germany \\ ${ }^{3}$ Technische Universität Dresden, D-01062 Dresden, Germany \\ ${ }^{4}$ Paul Scherrer Institut, CH-5232 Villigen, Switzerland \\ 5 Japan Atomic Energy Research Institute, Tokai, Ibaraki, 319-1195, Japan \\ ${ }^{6}$ Paul Scherrer Institut, CH-5232 Villigen and Departement für Chemie und Biochemie, CH-3012 Universität Bern, Switzerland \\ 7 Institute for Transuranium Elements, D-76125 Karlsruhe, Germany
}

(Received January 27, 2000; accepted in revised form March 9, 2000)

\section{Zirconium / Hafnium / Thorium / Rutherfordium / Fluoride complexation / \\ Anion and cation exchange chromatography}

Summary. The fluoride complexation of the group-4 elements $\mathrm{Zr}$, Hf and Rf, and of the pseudo-homolog Th, has been investigated in mixed $\mathrm{HNO}_{3} / \mathrm{HF}$ solutions by studying $K_{d}$ values on both cation exchange resins (CIX) and anion exchange resins (AIX) using the automated rapid chemistry apparatus ARCA. On the CIX, the four elements are strongly retained as cations below $10^{-3} \mathrm{M}$ HF. For $\mathrm{Zr}$ and $\mathrm{Hf}$, the decrease of the $K_{d}$ values due to the formation of fluoride complexes occurs between $10^{-3} \mathrm{M}$ HF and $10^{-2} \mathrm{M}$ HF. For Rf and Th, this decrease is observed at one order of magnitude higher HF concentrations. On the AIX, for $\mathrm{Zr}$ and $\mathrm{Hf}$, a rise of the $K_{d}$ values due to the formation of anionic fluoride complexes is observed between $10^{-3} \mathrm{M} \mathrm{HF}$ and $10^{-2} \mathrm{M} \mathrm{HF}$, i.e. in the same range of HF concentrations where the decrease of the $K_{d}$ values on the CIX is observed, yielding a consistent picture. For Rf and Th, on the AIX, no rise of the $K_{d}$ values is observed even if the HF concentration is increased up to $1 \mathrm{M}$. By varying the concentration of the counter ion $\mathrm{NO}_{3}{ }^{-}$which is competing for the binding sites on the AIX resin, it could be shown, nevertheless, that Rf does form anionic fluoride complexes. Apparently, there is a more specific competition of $\mathrm{NO}_{3}{ }^{-}$with respect to $\left[\mathrm{RfF}_{x}\right]^{(x-4)-}$ than with $\left[\mathrm{ZrF}_{y}\right]^{(y-4)-}$ and $\left[\mathrm{HfF}_{z}\right]^{(z-4)-}$.

\section{Introduction}

The first aqueous chemistry study of seaborgium ( $\mathrm{Sg}$, element 106) [1, 2] was performed recently with the Automated Rapid Chemistry Apparatus ARCA II. ${ }^{265} \mathrm{Sg}\left(T_{1 / 2}=\right.$ $\left.7.4_{-2.7}^{+3.3} s[3]\right)$ was eluted from a cation exchange resin (CIX, Aminex A6) in $0.1 \mathrm{M} \mathrm{HNO}_{3} / 5 \cdot 10^{-4} \mathrm{M} \mathrm{HF}$ which is typical for a group- 6 element. Due to the half-minute time span needed to prepare samples and to start counting [2], ${ }^{265} \mathrm{Sg}$ itself was not detected, but correlated mother-daughter decays of its descendant, 78-s ${ }^{261} \mathrm{Rf}$. Therefore, it was important that a HF concentration was selected that was insuf-

\footnotetext{
* Author for correspondence

(E-mail: jvKratz@vkcmzd.chemie.uni-mainz.de).
}

ficient to elute group-4 elements [2]. This had been demonstrated for $\mathrm{Zr}$ and $\mathrm{Hf}$ and was assumed for Rf.

Due to the HSAB (Hard Soft Acid Base) concept [4], hexavalent $\mathrm{Sg}$ shows a higher affinity to $\mathrm{F}^{-}$ions than any $4+$ ion. In this concept, ions are characterized by their hardness. Small, multiply charged ions are hard; big, singly charged ions are soft. Strong interactions between anions and cations occur either if both of them are hard (maximum of the ionic interaction) or if both of them are soft (maximum of the electronic overlap interaction). $\mathrm{Sg}^{6+}$ is both smaller and higher charged than $\mathrm{Rf}^{4+}$ and, therefore, it is the harder cation and will interact stronger with the hard fluoride anion. Thus, the tendency to form anionic fluoride complexes should increase from $\mathrm{Rf}$ to $\mathrm{Sg}$. So even if the $\mathrm{Rf}$ fluoride complexing behaviour is different from that of $\mathrm{Zr}$ and $\mathrm{Hf}$ and is more similar to that of the pseudo-homo$\log \mathrm{Th}$, it should stick to the CIX column even at higher HF concentrations than $5 \cdot 10^{-4} \mathrm{M}$.

In any case, it was of interest to verify experimentally that ${ }^{261} \mathrm{Rf}$, under the condition of the seaborgium experiments, is not eluted from the CIX column and can only appear in the seaborgium sample as a result of the $\alpha$ decay of ${ }^{265} \mathrm{Sg}$.

This verification of the $\mathrm{Rf}$ behaviour was the starting point for a detailed study of the Rf behaviour on both CIX and AIX. These data were related to data for the group 4 homologs $\mathrm{Zr}$ and $\mathrm{Hf}$ as well as to the actinide pseudohomolog Th.

Several experiments have been performed concerning the fluoride complexation of $\mathrm{Rf}$ by other groups within the last years. Szeglowski et al. [5] showed that Rf forms anionic fluoride complexes in 0.2 M HF that were retained on an anion exchange column. The extraction of fluoride complexes of the group 4 elements, Ti, Zr, Hf and Rf, into TiOA was reported by Kacher et al. [6]. This work presents some evidence for the extraction of $\mathrm{Rf}$ into TiOA from $0.5 \mathrm{M}$ HF. By selecting conditions such that the retention time is on the order of the nuclear half life, Pfrepper et al. [7] showed that, within the error bars, the $K_{d}$ value for $\mathrm{Rf}$ on an AIX in mixed $\mathrm{HNO}_{3} / 0.27 \mathrm{M} \mathrm{HF}$ solutions is identical 
with that of $\mathrm{Hf}$ and that the structure of the anionic complexes is $\left[\mathrm{MF}_{6}\right]^{2-}$ both for $\mathrm{Hf}$ and Rf. The aqueous chemistry of Rf is reviewed in Ref. [8].

\section{Definitions of $K_{d}$ values}

The distribution of a chemical compound between two phases is defined by the distribution coefficient $K_{d}$. The most simple definition in a static system is derived directly from the concentrations of the concerning species in both phases:

$$
K_{d}=\frac{c^{\prime}}{c^{\prime \prime}}
$$

where the indices mark the different phases. With this definition, the $K_{d}$ value is dimensionless.

The observables in all experiments of this paper were radioactive decays. Therefore, it is suitable to describe the distribution coefficient as a ratio of specific activities instead of concentrations. The applied systems were always heterogenic containing a liquid (aqueous) and a solid (exchange resin) phase. Then, the $K_{d}$ is calculated as follows:

$$
K_{d}=\frac{A_{s}}{A_{l}}=\frac{A_{s 0} V_{l}}{A_{l 0} m_{s}}
$$

with $A_{s}$ specific activity in the solid phase $[\mathrm{Bq} / \mathrm{g}], A_{l}$ specific activity in the liquid phase $[\mathrm{Bq} / \mathrm{ml}], A_{s 0}$ activity in the solid phase $[\mathrm{Bq}], A_{l 0}$ activity in the liquid phase [Bq], $m_{s}$ mass of the resin [g], $V_{l}$ volume of the solution [ml].

Thus, because the specific activity is either $\mathrm{Bq} / \mathrm{g}$ or $\mathrm{Bq} / \mathrm{ml}$, the dimension of the $K_{d}$ values is $\mathrm{ml} / \mathrm{g}$.

These definitions are based on the assumption that equilibrium is reached.

In a dynamic chromatography system, the $K_{d}$ is described as

$$
K_{d}=\left(t_{r}-t_{0}\right) \frac{V}{m_{s}}
$$

with $t_{r}$ retention time [s], $t_{0}$ dead time of the chromatography system due to the free column volume [s], $V$ flow rate of the liquid phase $[\mathrm{ml} / \mathrm{s}], m_{s}$ mass of the solid phase $[\mathrm{g}]$.

The dimension of the $K_{d}$ value is again $\mathrm{ml} / \mathrm{g}$. A prerequisite for a meaningful $K_{d}$ value is that equilibrium is reached for the applied flow rates. Equilibrium is verified if static and dynamic partition experiments yield the same results.

\section{Experimental}

\subsection{Tracer production}

\subsubsection{Production of ${ }^{95} \mathrm{Zr}$}

${ }^{95} \mathrm{Zr}$ was produced at the TRIGA reactor at Mainz in thermal-neutron induced fission of ${ }^{235} \mathrm{U}$. A ${ }^{235} \mathrm{U}$ target covered with an $\mathrm{Al}$ backing was irradiated. The Al backing was removed and dissolved in concentrated $\mathrm{HCl} .{ }^{95} \mathrm{Zr}$ was chemically separated from the fission products on an AIX column (Dowex 1x8, 200-400 mesh). The fission products were fed onto the column in $12 \mathrm{M} \mathrm{HCl}$, and $\mathrm{Zr}$ was eluted with $2 \mathrm{M} \mathrm{HCl}$.
Alternatively, a solution of ${ }^{95} \mathrm{Zr} /{ }^{95} \mathrm{Nb}$ in $0.5 \mathrm{M}$ oxalic acid was purchased from CIS Diagnostik GmbH, Dreieich. To obtain an oxalic-acid free solution it was evaporated to dryness several times in the presence of $\mathrm{H}_{2} \mathrm{O}_{2} / \mathrm{HCl}$ in a teflon crucible and taken up in $12 \mathrm{M} \mathrm{HCl}$.

\subsubsection{Production of ${ }^{175} \mathrm{Hf}$}

${ }^{175} \mathrm{Hf}$ was produced at the Philips Cyclotron at Karlsruhe in the reaction ${ }^{175} \mathrm{Lu}(\mathrm{p}, \mathrm{n}){ }^{175} \mathrm{Hf}$. $500 \mathrm{mg} \mathrm{Lu}$ oxide were pressed in between two $1.1 \mathrm{~mm}$ graphite disks. This target was irradiated for one hour with a $10 \mu \mathrm{A} 20 \mathrm{MeV}$ proton beam, resulting in $16 \mathrm{MeV}$ protons entering the target. These conditions were chosen such that the protons were fully stopped in the target material. Thus the energy of the protons covered a large portion of the exitation function for the $(\mathrm{p}, \mathrm{n})$ reaction. The exitation function was calculated with the HIVAP code [9]. After irradiation, the target material was dissolved in concentrated $\mathrm{HCl}$. Lu was removed on an AIX column (Dowex 1x8, 200-400 mesh) from which the ${ }^{175} \mathrm{Hf}$ was subsequently eluted with $4 \mathrm{M} \mathrm{HCl}$.

\subsubsection{Production of ${ }^{233} \mathrm{Th}$}

${ }^{233} \mathrm{Th}$ was produced by neutron irradiation of ${ }^{232} \mathrm{Th}$ at the TRIGA reactor Mainz. The Th results are the only data described within this work that were obtained with non carrier-free isotopes. The irradiation times were chosen such that the carrier amount in the used solutions was about a factor of 100 lower than the loading capacity of the exchanger material.

\subsection{Batch experiments}

All tracer solutions for batch experiments were stored in $12 \mathrm{M} \mathrm{HCl}$ in polypropylene flasks. Batches of 100 to $200 \mathrm{mg}$ of ion-exchange resin and 2 to $4 \mathrm{ml}$ acid solution were shaken for about 20 minutes. After centrifuging, half of the aqueous phase was measured by $\gamma$-spectroscopy. The activity of an equal amount of the genuine tracer solution was also measured. The activity of the solid phase was calculated as the difference of both activities and the $K_{d}$ values were calculated using Eq. (2).

Hence, geometric effects as they would result in the direct measurement of the exchanger resin, could be avoided. No wall-adsorption effects were observed.

\subsection{On-line experiments}

\subsubsection{Rf production and transport}

${ }^{261} \mathrm{Rf}$ was produced directly in the reaction ${ }^{248} \mathrm{Cm}\left({ }^{18} \mathrm{O}, 5 \mathrm{n}\right){ }^{261} \mathrm{Rf}$ at the Philips Cyclotron of the Paul Scherrer Institute (PSI) Villigen, Switzerland.

The $730 \mu \mathrm{g} / \mathrm{cm}^{2}{ }^{248} \mathrm{Cm}$ target ${ }^{1}$ was prepared by electrodeposition of $\mathrm{Cm}\left(\mathrm{NO}_{3}\right)_{3}$ on $\mathrm{Be}$. After the plating process, the nitrate was converted into the oxide by heating. It was

${ }^{1} 871 \mu \mathrm{g} / \mathrm{cm}^{2}{ }^{248} \mathrm{Cm}$ oxide plus $96 \mu \mathrm{g} / \mathrm{cm}^{2} \mathrm{Gd}$ oxide of a mean mass $\mathrm{m}_{G d}=155 \mathrm{amu}$. 
bombarded with average ${ }^{18} \mathrm{O}^{5+}$ beam intensities of $3 \cdot 10^{12} \mathrm{~s}^{-1}$.

The $128.6(133)^{2} \mathrm{MeV}$ ion beam passed through a 10 (12.5) $\mu \mathrm{m}$ Havar window, $4 \mathrm{~mm} \mathrm{~N}_{2}$ cooling gas and the $12.7 \mu \mathrm{m}$ Be backing entering the target material with a projectile energy of $100.3(100.2) \mathrm{MeV}$, and leaving the target material with 98.7 (98.6) $\mathrm{MeV}$. This corresponds to the maximum of the excitation function [9]. A production cross section of approximately $5 \mathrm{nb}$ is observed in the ${ }^{248} \mathrm{Cm}\left({ }^{18} \mathrm{O}, 5 \mathrm{n}\right)$ reaction close to the maximum of the excitation function [10]. This results in a production rate of about 2 atoms/min under the given conditions.

The target contained $10 \% \mathrm{Gd}^{3}$ to produce simultaneously short-lived Hf isotopes that were used to monitor on-line the behaviour of $\mathrm{Hf}$ and to perform yield checks by $\gamma$-spectroscopy. Reaction products recoiling out of the target were stopped in $\mathrm{He}$ gas containing $\mathrm{KCl}$ aerosols generated by sublimation from solid $\mathrm{KCl}$ at $640^{\circ} \mathrm{C}$ [8]. The aerosols together with the recoil products were transported through a steel capillary $(2 \mathrm{~mm}$ inner diameter, length $50 \mathrm{~m}$ ) to the automated chemistry apparatus ARCA II [11]. At a flow rate of about $2.5 \mathrm{l} / \mathrm{min} \mathrm{He}$, this leads to a transport yield of about $50 \%$.

In ARCA, the $\mathrm{He}$ is pumped away while the $\mathrm{KCl}$ clusters are collected by impaction on the top slider of ARCA II [12].

\subsubsection{On-line production of short-lived Hf isotopes}

The short lived Hf isotopes ${ }^{166,167,168, \ldots} \mathrm{Hf}$ were produced with the ${ }^{18} \mathrm{O}$ beam at the Philips Cyclotron at PSI in the xnreactions using the $\mathrm{Gd}$ content of the $\mathrm{Cm}$ target or at the GSI UNILAC in the xn-reactions of ${ }^{12} \mathrm{C}$ with an enriched ${ }^{158}$ Dy target. The targets were irradiated with a $100 \mathrm{MeV}$ ${ }^{18} \mathrm{O}$ beam, populating mostly the $5 \mathrm{n}$ channel, or with a $95 \mathrm{MeV}{ }^{12} \mathrm{C}$ beam, respectively, populating the $4 \mathrm{n}$ and $5 \mathrm{n}$ channel (energies are corrected for the energy loss in the entrance window, cooling gas and target backing). The recoil products were collected as the Rf recoils described above.

\subsection{Automated HPLC with Rf and Hf produced on-line}

\subsubsection{Rf experiments with the CIX}

Rf and $\mathrm{Hf}$ were transported by a $\mathrm{He}(\mathrm{KCl})$ gas jet and collected for $90 \mathrm{~s}$ by impaction on the slider in ARCA II [11]. The deposit was dissolved in $200 \mu \mathrm{l} 0.1 \mathrm{M} \mathrm{HNO}_{3} / \mathrm{x} \mathrm{M} \mathrm{HF}$ (x varied) and fed onto the $1.6 \times 8 \mathrm{~mm}$ cation-exchange column $^{4}$ at a flow rate of $1 \mathrm{ml} / \mathrm{min}$. The column had been conditioned with $500 \mu \mathrm{l}$ of the feeding solution. The effluent was evaporated to dryness as sample 1 . In order to elute remaining $\mathrm{Rf}$ and $\mathrm{Hf}$ from the column, a second fraction of $200 \mu \mathrm{l} 0.1 \mathrm{M} \mathrm{HNO}_{3} / 0.1 \mathrm{M} \mathrm{HF}$ was collected to strip all

\footnotetext{
2 The values given in parenthesis refer to the conditions when a thicker Havar window was chosen.

${ }^{3}$ Isotopic composition $26.2 \%{ }^{152} \mathrm{Gd}, 9.8 \%{ }^{154} \mathrm{Gd}, 22.3 \%{ }^{155} \mathrm{Gd}$, $16.5 \%{ }^{156} \mathrm{Gd}, 8.3 \%{ }^{157} \mathrm{Gd}, 10.2 \%{ }^{158} \mathrm{Gd}, 6.7 \%{ }^{160} \mathrm{Gd}$.

${ }^{4}$ BioRad Aminex A6 in the $\mathrm{H}^{+}$form, an exchange resin of the AG50WX8 type, produced especially for HPLC with a particle size of $17.5 \pm 2 \mu \mathrm{m}$.
}

group 4 elements from the column. This fraction was prepared as sample 2 .

\subsubsection{Rf experiments with the AIX}

Rf and Hf were transported, collected and loaded onto the anion-exchange column ${ }^{5}$ as in the experiments with the CIX. Again, the effluent was evaporated to dryness as sample 1. In order to elute remaining $\mathrm{Rf}$ and $\mathrm{Hf}$ from the column, a second fraction of $200 \mu \mathrm{l} 5 \mathrm{M} \mathrm{HNO}_{3} / 0.01 \mathrm{M} \mathrm{HF}$ was used. This fraction was prepared as sample 2.

\subsubsection{Detection of $\mathrm{Rf}$}

78-s ${ }^{261} \mathrm{Rf}$ was detected by $\alpha$-spectroscopy using sixteen $450 \mathrm{~mm}-2$ passivated implanted planar silicon (PIPS) detectors. At a given cycle time of $90 \mathrm{~s}$ and the possibility to measure 8 pairs of samples, the counting time for each sample was $12 \mathrm{~min}$. List mode data were stored for each $\alpha$ decay, including energy, detector number and time after start of the measurement. The $\alpha$-energy resolution was about $30 \mathrm{keV}$ for $\alpha$-particles between 5 and $10 \mathrm{MeV}$.

The detection efficiency for an $\alpha$ particle was $30 \%$ thus being $9 \%$ for $\alpha \alpha$ correlations detecting both the decay of ${ }^{261} \mathrm{Rf}$ and its daughter ${ }^{257}$ No. These efficiencies lead to an expected ratio of $4.67: 1$ between $\alpha$ singles and $\alpha \alpha$ correlations ${ }^{6}$.

In the Rf experiments, every 8th pair of samples was additionally monitored by $\gamma$-spectroscopy to determine the distribution of $\mathrm{Hf}$ and its chemical yield.

The $K_{d}$ values were calculated using Eq. (2) such that the activity of sample 1 was attributed to the aqueous phase, the activity of sample 2 was attributed to the solid phase. The amount of exchange resin $(6.6 \mathrm{mg})$ was determined from the average weight of 20 used columns. The number of $\alpha$ counts in fraction 2 was corrected for the decay occurring between the elution of fraction 1 and 2 . The effluent volume was corrected for the dead volume of ARCA $(35 \mu \mathrm{l})$.

In the AIX chemistry, the cations are expected to run through the column. Therefore, the ${ }^{261} \mathrm{Rf}$ daughter ${ }^{257} \mathrm{No}$ formed during the collection time before the chemical separation ("primordial No") has to be taken into account. (The distribution of No formed after the chemical separation is characteristic of the behaviour of its mother nuclide Rf).

As can be calculated from the equations of radioactive decay and growth, the contribution of the "primordial No" activity to the measured $\alpha$-singles is $4.6 \%$. The distribution of these No events between the two fractions was assumed to be modeled by the behaviour of the actinide Fm represented by the 6.9 to $7.2 \mathrm{MeV}$ group in the $\alpha$-particle spec$\operatorname{tra}^{7}$.

\footnotetext{
${ }^{5}$ Riedel-de Haën in the chloride form, an ion-exchange resin similar to the BioRad AG1X8 type, produced especially for HPLC with a particle size of $26 \pm 6 \mu \mathrm{m}$.

$6100 \mathrm{Rf}$ decays cause 60 measured events, i.e. 9 correlations, 21 decays missing the daughter decay and 21 events missing the mother decay, assuming a time window open infinitely long.

It should be noticed that the effective correction for the "primordial" No becomes almost negligible if the $K_{d}$ values are relatively low.
} 


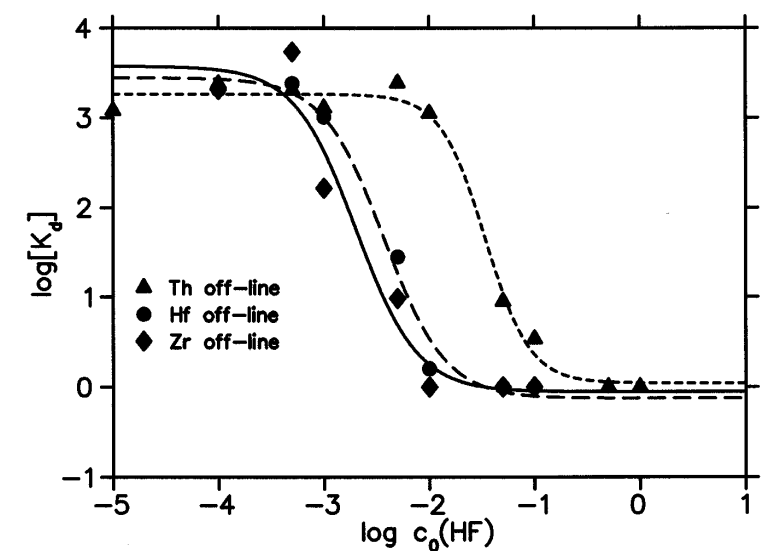

Fig. 1. Sorption of $\mathrm{Zr}$, Hf and $\mathrm{Th}$ (in batch experiments) on the CIX resin (Aminex A6) in $0.1 \mathrm{M} \mathrm{HNO}_{3}$ at various $\mathrm{HF}$ concentrations. The lines are tanh functions fitted to the data points.

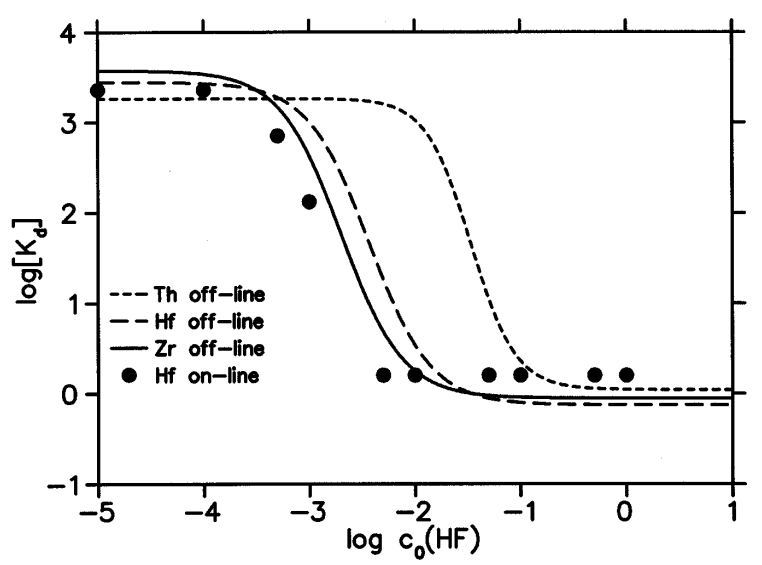

Fig. 2. Sorption of $\mathrm{Hf}$ (produced on-line) on the CIX resin (Aminex A6) in $0.1 \mathrm{M} \mathrm{HNO}_{3}$ at various $\mathrm{HF}$ concentrations. The lines are tanh functions fitted to the off-line data points (shown in Fig.1).

\section{Results}

\subsection{Tracer experiments}

\subsubsection{Behaviour of $\mathrm{Zr}$, Hf and Th on the CIX}

The results of the batch tracer experiments on the CIX are given in Fig. 1. In $0.1 \mathrm{M} \mathrm{HNO}_{3}$, all elements stick to the cation-exchange resin at $\mathrm{HF}$ concentrations $<10^{-3} \mathrm{M}$, indicating that, at low HF concentrations, cationic species are formed and are held back very strongly on the resin with $\log \left(K_{d}\right)$ values $>3$. In a concentration range between $10^{-3} \mathrm{M}<[\mathrm{HF}]<10^{-2} \mathrm{M}$, the $K_{d}$ values of the group 4 elements decrease. At these concentrations, neutral or anionic fluoride complexes may be formed. The behaviour of $\mathrm{Zr}$ and $\mathrm{Hf}$ is very similar (Hf sticking to the resin even at slightly higher HF concentrations). This is in agreement with the known general similarities of these elements. The elution of Th from the column is observed at about 1 order of magnitude higher HF concentrations.

The data taken for Hf on-line (short lived isotopes measured with ARCA) are in full agreement with the offline (batch) data (Fig. 2), indicating that at the flow rate

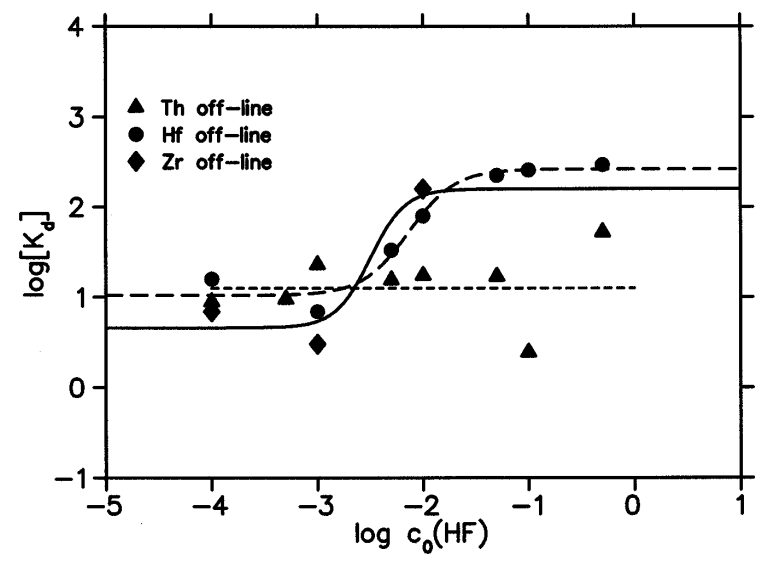

Fig. 3. Sorption of $\mathrm{Zr}$, $\mathrm{Hf}$ and $\mathrm{Th}$ (in batch experiments) on the AIX resin (Riedel-de Haën) in $0.1 \mathrm{M} \mathrm{HNO}_{3}$ at various HF concentrations. The lines are tanh functions fitted to the data points.

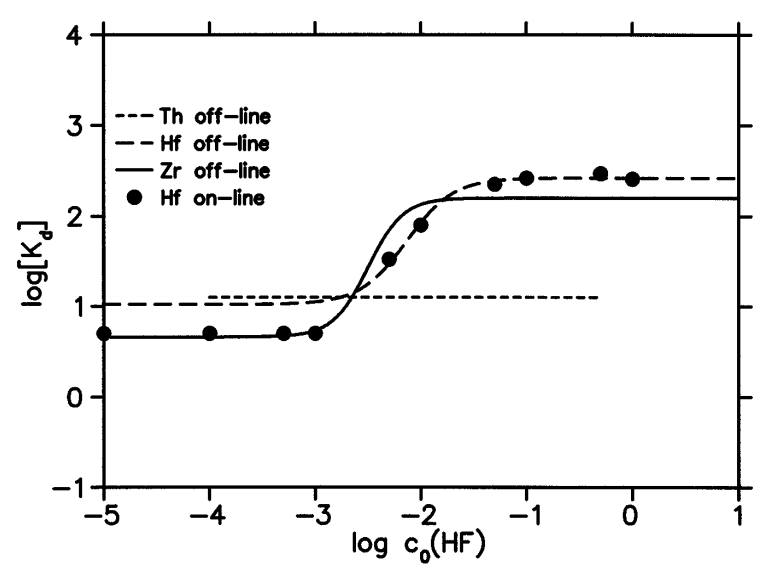

Fig. 4. Sorption of Hf (produced on-line) on the AIX resin (Riedel-de Haën) in $0.1 \mathrm{M} \mathrm{HNO}_{3}$ at various HF concentrations. The lines are tanh functions fitted to the off-line data points (shown in Fig. 3).

of $1 \mathrm{ml} / \mathrm{min}$ equilibrium is reached in the column experiments.

\subsubsection{Behaviour of $\mathrm{Zr}$, Hf and Th on the AIX}

The results of the tracer experiments on the AIX are given in Fig. 3. As is expected, at low HF concentrations, all elements are running through the column. In a concentration range of about $10^{-3} \mathrm{M}<[\mathrm{HF}]<10^{-2} \mathrm{M}$, the $K_{d}$ values of $\mathrm{Zr}$ and $\mathrm{Hf}$ rise simultaneously, i.e. in the same range as they decrease on the CIX. This shows clearly, that the disappearance of the cationic species at low HF concentrations is followed immediately by the formation of anionic complexes.

For Th, no anionic species are known. This results in the neutral $\mathrm{ThF}_{4}$ being the final compound. Hence Th is eluted from the AIX at all HF concentrations. The scattering of the Th data in the high HF concentration range might be attributed to the usage of non-carrier free Th. The data taken for Hf on-line (measured with ARCA) are consistent with the off-line (batch) data (Fig. 4), indicating that at the flow rate of $1 \mathrm{ml} / \mathrm{min}$ equilibrium is reached in the column experiments. 


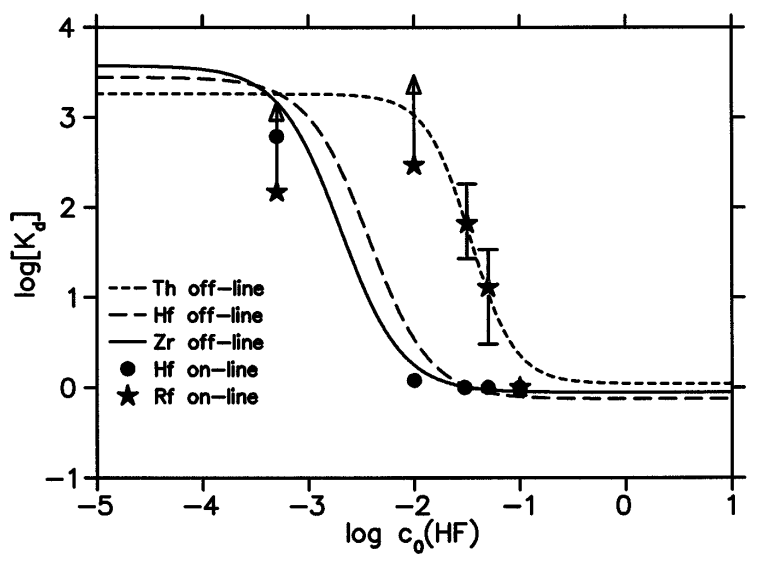

Fig. 5. Sorption of $\mathrm{Zr}$, Hf, Th, and Rf on the CIX resin (Aminex A6) in $0.1 \mathrm{M} \mathrm{HNO}_{3}$ at various $\mathrm{HF}$ concentrations. The data points of $\mathrm{Hf}$ and $\mathrm{Rf}$ are taken from the on-line experiments. The lines are tanh functions fitted to the off-line data points. The different error bars are described in the text.

\subsection{Rutherfordium experiments}

In more than 3000 experiments 92 single events and 18 correlations were detected in the $E_{\alpha}$ window between 8.1 and $8.5 \mathrm{MeV}$ that could be attributed to the decay of ${ }^{261} \mathrm{Rf}$ and/or the decay of its daughter ${ }^{259}$ No. From the 61 single events in the AIX chemistry, 3 are considered to be decays of "primordial" No. This results in an overall single/correlation ratio of $4.9: 1$. Thus within the statistical error, this number is in full agreement with the expected ratio of 4.67:1 determined by the detector geometry (see above).

\subsubsection{Behaviour of $\mathrm{Rf}$ on the CIX}

The results of the on-line experiments with the CIX are given in Fig. 5. It was shown experimentally that ${ }^{261} \mathrm{Rf}$ is not eluted from the CIX column under the conditions of the previously performed $\mathrm{Sg}$ experiment and can only appear in the $\mathrm{Sg}$ sample as a result of the decay of ${ }^{265} \mathrm{Sg}$. The measured $K_{d}(\mathrm{Rf})$ was $>148$ at $5 \cdot 10^{-4} \mathrm{M}$ HF. Further, the distribution coefficient of ${ }^{261} \mathrm{Rf}$ on a cation exchange resin at a higher $\mathrm{HF}$ concentration $\left(0.1 \mathrm{M} \mathrm{HNO}_{3} / 0.01 \mathrm{M} \mathrm{HF}\right)$ was studied. At this concentration, despite $\mathrm{Zr}$ and $\mathrm{Hf}$ being eluted, $\mathrm{Rf}$ is still retained on the column (Fig. 5). The $K_{d}$ (Rf) is $>312$ at $10^{-2} \mathrm{M} \mathrm{HF}$.

These $K_{d}$ values are lower limits, because no Rf events were detected in fraction 1 . In order to define these lower limits, a Monte Carlo simulation was performed to calculate which number of events would be in agreement with a measured number of 0 at a significance level of $90 \%$. There is no reason why the $K_{d}$ at higher HF concentrations should be higher than at $5 \cdot 10^{-4} \mathrm{M} \mathrm{HF}$, so it could be concluded that the $K_{d}$ at any concentration $<0.01 \mathrm{M} \mathrm{HF}$ should be $>312$. The $\log \left(K_{d}(\mathrm{Rf})\right)$ in the strip fraction $(0.1 \mathrm{M} \mathrm{HF})$ was fixed at 0 assuming that the behaviour of $\mathrm{Rf}$ is the same as that of $\mathrm{Zr}$ and $\mathrm{Hf}$ at this concentration. The number of measured events is in agreement with the number of expected events calculated from the known cross section.

The different behaviour of $\mathrm{Rf}$ as compared to that of $\mathrm{Zr}$ and $\mathrm{Hf}$ was felt to deserve further investigation. Therefore, the $K_{d}$ values of $\mathrm{Rf}$ at $\mathrm{HF}$ concentrations of $0.03 \mathrm{M}$ and

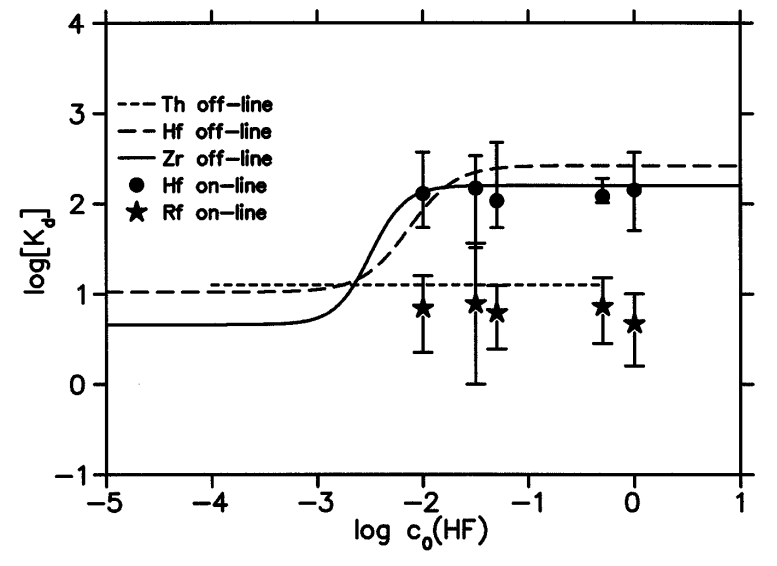

Fig. 6. Sorption of $\mathrm{Zr}$, Hf, Th, and $\mathrm{Rf}$ on the AIX resin (Riedel-de $\mathrm{Haën}$ ) in $0.1 \mathrm{M} \mathrm{HNO}_{3}$ at various $\mathrm{HF}$ concentrations. The data points of $\mathrm{Hf}$ and $\mathrm{Rf}$ are taken from the on-line experiments. The lines are tanh functions fitted to the off-line data points.

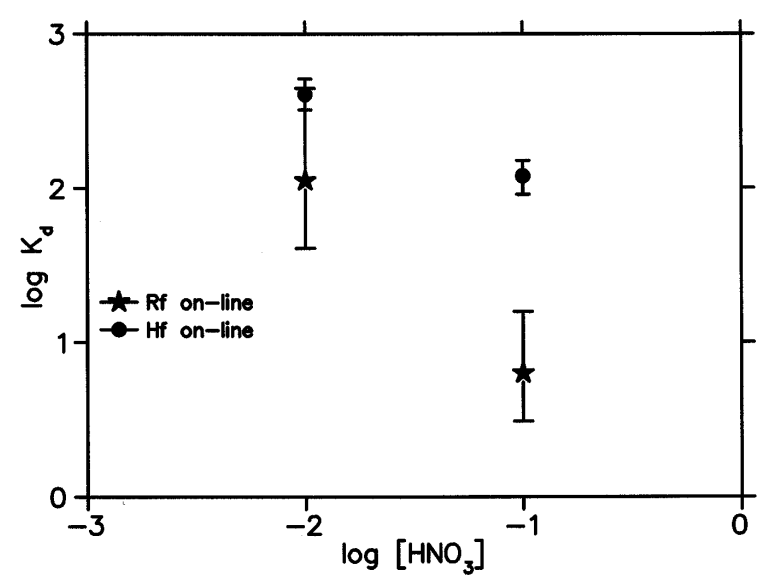

Fig. 7. Evidence for the existence of negatively charged Rf Fluoride complexes in $0.05 \mathrm{M} \mathrm{HF}$. Hf and Rf data points are taken from online experiments.

$0.05 \mathrm{M}$ (both in $0.1 \mathrm{M} \mathrm{HNO}_{3}$ ) were determined in order to establish at which concentration the $K_{d}$ decreases indicating that neutral or anionic complexes of Rf are being formed (Fig. 5). On the CIX, the decrease of the $K_{d}$ values for Rf occurs between $0.01 \mathrm{M}$ and $0.1 \mathrm{M} \mathrm{HF}$, i.e. at one order of magnitude higher $\mathrm{HF}$ concentrations than for $\mathrm{Zr}$ and $\mathrm{Hf}$. Under the given conditions, $\mathrm{Rf}$ seems to resemble rather its pseudo-homolog Th than its group members $\mathrm{Zr}$ and $\mathrm{Hf}$.

\subsubsection{Behaviour of $\mathrm{Rf}$ on the AIX}

The results of the on-line experiments with the AIX are given in Fig. 6. It was expected that Rf would form anionic complexes at high HF concentrations resulting in high $K_{d}$ values, while $\mathrm{Th}$, which does not form anionic fluoride complexes, should have low $K_{d}$ values. To our surprise, the data show that $\mathrm{Rf}$ behaves differently from $\mathrm{Zr}$ and $\mathrm{Hf}$ and that it seems to resemble Th also on the AIX. Even for $1 \mathrm{M}$ HF which is about 2 orders of magnitude higher than the concentration from whereon maximum $K_{d}$ values are observed for $\mathrm{Zr}$ and $\mathrm{Hf}$, there is no indication of a rise of the $K_{d}$ value for Rf. 
Table 1. Concentrations of different species in $\mathrm{HNO}_{3} / \mathrm{HF}$ media calculated from $p K_{s}(\mathrm{HF})=2.85$.

\begin{tabular}{lllll}
\hline$\left[\mathrm{HNO}_{3}\right]$ & {$[\mathrm{HF}]_{0}$} & {$\left[\mathrm{~F}^{-}\right]$} & {$\left[\mathrm{H}^{+}\right]$} & {$[\mathrm{HF}]$} \\
\hline 0.1 & 0.03 & 0.00021 & 0.1002 & 0.0298 \\
0.1 & 0.05 & 0.00035 & 0.1003 & 0.0497 \\
0.1 & 0.1 & 0.0007 & 0.1007 & 0.0993 \\
0.1 & 0.3 & 0.0021 & 0.1021 & 0.30 \\
0.1 & 0.5 & 0.0034 & 0.1034 & 0.50 \\
0.1 & 1.0 & 0.0067 & 0.1067 & 0.99 \\
0.01 & 0.03 & 0.0016 & 0.0116 & 0.0284 \\
0.01 & 0.05 & 0.0026 & 0.0126 & 0.0474 \\
0.01 & 0.1 & 0.0048 & 0.0148 & 0.0952 \\
0.01 & 0.3 & 0.012 & 0.0216 & 0.29 \\
0.01 & 0.5 & 0.017 & 0.0269 & 0.48 \\
0.01 & 1.0 & 0.027 & 0.0372 & 0.97 \\
\hline
\end{tabular}

Table 2. Ionic radii of $\mathrm{Zr}$, Hf, Rf and Th.

\begin{tabular}{lllll}
\hline Ion & $\mathrm{Zr}^{4+}$ & $\mathrm{Hf}^{4+}$ & $\mathrm{Rf}^{4+}$ & $\mathrm{Th}^{4+}$ \\
\hline Ionic radius, ( $\mathrm{\AA})$ & $0.72^{a}$ & $0.71^{a}$ & $0.79^{b}$ & $1.08^{b}$ \\
\hline
\end{tabular}

a: [14]; b: [15].

As it would be in contradiction to the results of Refs. [5-7] that there are no anionic fluoride complexes formed for $\mathrm{Rf}$ at any $\mathrm{HF}$ concentration, the concentration of $\mathrm{HNO}_{3}$ with $\mathrm{NO}_{3}{ }^{-}$serving as a counter ion competing for the binding sites on the anion-exchange resin was varied from 0.1 to 0.01 at a constant $\mathrm{HF}$ concentration of $0.05 \mathrm{M}$ in an additional experiment. By plotting $\log \left(K_{d}\right)$ vs. $\log$ $\left(\mathrm{c}\left(\mathrm{NO}_{3}{ }^{-}\right)\right)$one expects to obtain a linear relation with a slope corresponding to the charge of the complex [13].

As is shown in Fig. 7, in this experiment, qualitative evidence for negatively charged complexes of $\mathrm{Hf}$ and $\mathrm{Rf}$ was observed as in both cases, the $K_{d}$ value rises with decreasing $\mathrm{NO}_{3}{ }^{-}$concentration. Due to the large uncertainties, the ionic charge state cannot be determined unambiguously from the present data. One might argue that the analysis of $\log \left(K_{d}\right) v s . \log \left(\mathrm{c}\left(\mathrm{NO}_{3}{ }^{-}\right)\right)$must be done at a constant free fluoride concentration rather than at constant HF concentration. According to Table 1, the same $\mathrm{F}^{-}$concentration as in $0.01 \mathrm{M} \mathrm{HNO}_{3} / 0.05 \mathrm{M} \mathrm{HF}$ is present in $0.1 \mathrm{M} \mathrm{HNO}_{3}$ if the $\mathrm{HF}$ concentration is chosen between $0.3 \mathrm{M}$ and $0.5 \mathrm{M}$ HF. As the $K_{d}$ values for Rf in $0.1 \mathrm{M} \mathrm{HNO}_{3}$ are all the same for $\mathrm{HF}$ concentrations between $0.01 \mathrm{M}$ and $1 \mathrm{M}$ within the uncertainties, a figure literally indistinguishable from Fig. 7 would result if a constant $\mathrm{F}^{-}$concentration were chosen as the condition. Fig. 7 gives the hint that it is the action of the counter ion $\mathrm{NO}_{3}{ }^{-}$which is responsible for the different behavior of $\mathrm{Rf}$ as compared to $\mathrm{Zr}$ and $\mathrm{Hf}$.

\section{Discussion}

We have observed properties of $\mathrm{Rf}$ in $0.1 \mathrm{M} \mathrm{HNO}_{3}$ in the presence of variable HF concentrations that deviate significantly from those of its homologs $\mathrm{Zr}$ and $\mathrm{Hf}$ and resemble those of Th.

On the CIX columns, the $K_{d}$ values for $\mathrm{Zr}$ and $\mathrm{Hf}$ decrease between $10^{-3}$ and $10^{-2} \mathrm{M}$ HF due to the formation of fluoride complexes. For Rf and Th, this decrease occurs at one order of magnitude higher HF concentrations. A qualitative interpretation from the HSAB point of view is possible by using the ionic radii contained in Table 2 . The radii of $\mathrm{Zr}^{4+}$ and $\mathrm{Hf}^{4+}$ are very similar due to the lanthanide contraction resulting in a similar complexation behaviour. As the hardness as electron acceptors correlates with the size of the ions it will decrease in the order $\mathrm{Zr}^{4+} \approx \mathrm{Hf}^{4+}>$ $\mathrm{Rf}^{4+}>\mathrm{Th}^{4+}$. The fluoride anion is a hard anion and interacts stronger with small (hard) cations. From this, one would expect a weaker fluoride complexation of Rf as compared to $\mathrm{Zr}$ and $\mathrm{Hf}$ as experimentally observed. However, the apparent coincidence of the $\operatorname{Rf} K_{d}$ values with those of $\mathrm{Th}$ is unexpected.

On the AIX columns, the absence of a rise of the $K_{d}$ values of $\mathrm{Rf}$ is even more surprising, and a theoretical explanation is not at hand at this time. Very informative was the variation of the concentration of the counter ion $\mathrm{NO}_{3}{ }^{-}$ which gave the hint that, at the $\mathrm{F}^{-}$concentration of about $3 \cdot 10^{-3} \mathrm{M}$, Rf does form anionic fluoride complexes, however, the competition of the counter ion $\mathrm{NO}_{3}^{-}$for the binding sites on the anion-exchange resin seems to be more effective with respect to the $\mathrm{Rf}$ complex than with respect to the $\mathrm{Zr}$ and $\mathrm{Hf}$ complexes.

To shed more light on the remaining open questions, additional experiments are necessary in which i) the $\mathrm{NO}_{3}{ }^{-}$ concentration is varied systematically at a given fluoride concentration and ii) the fluoride concentration is varied systematically at a significantly lower $\mathrm{NO}_{3}{ }^{-}$concentration (e.g. $0.01 \mathrm{M} \mathrm{HNO}_{3}$ ) than in the present work.

Acknowledgements. We wish to thank the staff and crew of the Philips Cyclotron at the PSI for providing the ${ }^{18} \mathrm{O}$ beam. The assistance of $\mathrm{R}$. Eichler, A. Grund and M. Gärtner during some of the PSI experiments is highly appreciated. Thanks are due to the Karlsruhe Philips Cyclotron staff for irradiating the Lu targets.

\section{References}

1. Schädel, M., Brüchle, W., Dressler, R., Eichler, B., Gäggeler, H.W., Günther, R., Gregorich, K.E., Hoffman, D.C., Hübener, S., Jost, D.T., Kratz, J.V., Paulus, W., Schumann, D., Timokhin, S., Trautmann, N., Türler, A., Wirth, G., Yakushev, A.: Nature 388, 55 (1997).

2. Schädel, M., Brüchle, W., Dressler, R., Eichler, B., Gäggeler, H.W., Schausten, B., Schimpf, E., Jäger, E., Wirth, G., Günther, R., Kratz, J.V., Paulus, W., Seibert, A., Thörle, P., Trautmann, N., Zauner, S., Schumann, D., Andrassy, M., Misiak, R., Gregorich, K.E., Hoffman, D.C., Lee, D.M., Sylwester, E.R., Nagame, Y., Oura, Y.: Radiochim. Acta 77, 149 (1997).

3. Türler, A., Dressler, R., Eichler, B., Gäggeler, H. W., Jost, D. T., Schädel, M., Brüchle, W., Gregorich, K. E., Trautmann, N., Taut, S. : Phys. Rev. C57, 1648 (1998).

4. Pearson, R. G.: J. Am. Chem. Soc. 85, 3533 (1963).

5. Szeglowski, Z., Bruchertseifer, H., Domanov, V. P., Gleisberg, B., Guseva, L. J., Hussonnois, M., Tikhomirova, G. S., Zvara, I., Oganessian, Yu. Ts.: Radiochim. Acta 51, 71 (1990).

6. Kacher, C. D., Gregorich, K. E., Lee, D. M., Watanabe, Y., Kadkhodayan, B., Wierczinski, B., Lane, M. R., Sylwester, E. R, Keeney, D. A., Hendriks, M., Hoffman, D. C., Bilewicz, A.: Radiochim. Acta 75, 135 (1996).

7. Pfrepper, G., Pfrepper, R., Krauss, D., Yakushev, A. B., Timokhin, S. N., Zvara, I.: Radiochim. Acta 80, 7 (1998).

8. Kratz, J. V.: in: Heavy Elements and Related New Phenomena, ed. Greiner, W., Gupta, R. K., World Scientific, Singapore, 129193 (1999).

9. Reisdorf, W.: Z. Phys. A - Atoms and Nuclei 300, 227 (1981). 
10. Ghiorso, A., Nurmia, M., Eskola, K., Eskola, P.: Phys. Lett. 32 B, 95 (1970).

11. Schädel, M., Brüchle, W., Jäger, E., Schimpf, E., Kratz, J. V., Scherer, U. W., Zimmermann, P.: Radiochim. Acta 48, 171 (1989).

12. Paulus, W.: doctoral thesis, Mainz (1997).
13. Adloff, J. P., Guillaumont, R.: Fundamentals of Radiochemistry, CRC Press, Boca Raton (1993).

14. Shannon, R. D.: Acta Crystallogr. Sect. A 32, 751 (1976).

15. Johnson, E., Fricke, B., Keller, O. L., Jr., Nestor, C. W., Jr., Tucker, T. C.: J. Chem. Phys. 93, 8041 (1990). 